\title{
RESULTS ON THE BETA FUNCTION
}

\author{
BRIAN FISHER
}

\section{INTRODUCTION}

The Beta function is usually defined by

$$
B(x, y)=\int_{0}^{1} t^{x-1}(1-t)^{y-1} d t
$$

for $x, y>0$, see for example Sneddon [4]. It then follows that

$$
B(x, y)=\frac{\Gamma(x) \Gamma(y)}{\Gamma(x+y)}
$$

and this equation is used to define $B(x, y)$ for $x, y<0$ and $x, y \neq-1,-2, \ldots$

It was proved in [2] that

$$
B(x, y)=\mathrm{N}-\lim _{\epsilon \rightarrow 0} \int_{\epsilon}^{1-\epsilon} t^{x-1}(1-t)^{y-1} d t
$$

for $x, y \neq 0,-1,-2, \ldots$, where $N$ is the neutrix, see van der Corput [1], having the domain $N^{\prime}=\left\{\epsilon: 0<\epsilon<\frac{1}{2}\right\}$ with negligible functions finite linear sums of the functions

$$
\epsilon^{\lambda} \ln ^{r-1} \epsilon, \quad \ln ^{r} \epsilon \quad(\lambda<0, \quad r=1,2, \ldots)
$$

and all functions of $\epsilon$ which converge to zero in the usual sense as $\epsilon$ tends to zero.

Note that if a function $F(\epsilon)=\nu(\epsilon)+f(\epsilon)$, where $\nu(\epsilon)$ is the sum of the divergent negligible functions of $F(\epsilon)$, then p.f. $F(\epsilon)$, Hadamard's finite part of $F(\epsilon)$, is equal to $f(\epsilon)$ and so

$$
\mathrm{N}-\lim _{\epsilon \rightarrow 0} F(\epsilon)=\lim _{\epsilon \rightarrow 0} f(\epsilon)=\lim _{\epsilon \rightarrow 0} \text { p.f. } F(\epsilon) .
$$

Thus, taking the neutrix limit of a function $F_{n}(\epsilon)$ as $\epsilon$ tends to 0 is equivalent to taking the normal limit of the function p.f. $F(\epsilon)$ as $\epsilon$ tends to 0 . 
It was proved that this neutrix limit for $B(x, y)$ exists for all $x, y$ and so was used to define $B(x, y)$ for all $x, y$. Note that if $x>0$, we could write

$$
B(x, y)=\mathrm{N}-\lim _{\epsilon \rightarrow 0} \int_{0}^{1-\epsilon} t^{x-1}(1-t)^{y-1} d t
$$

and if $y>0$, we could write

$$
B(x, y)=\mathrm{N}-\lim _{\epsilon \rightarrow 0} \int_{\epsilon}^{1} t^{x-1}(1-t)^{y-1} d t .
$$

For example, it was proved in [2] that

$$
B(0,0)=B(0,1)=0
$$

and

$$
B(0, r)=\sum_{i=1}^{r-1}\left(\begin{array}{c}
r-1 \\
i
\end{array}\right) \frac{(-1)^{i}}{i+1}
$$

for $r=1,2, \ldots$.

More generally, it was proved in [3] that if

$$
B_{p, q}(x, y)=\frac{\partial^{p+q}}{\partial x^{p} \partial x^{q}} B(x, y),
$$

then

$$
B_{p, q}(x, y)=\mathrm{N}-\lim _{\epsilon \rightarrow 0} \int_{\epsilon}^{1-\epsilon} t^{x-1} \ln ^{p} t(1-t)^{y-1} \ln ^{q}(1-t) d t
$$

for $x, y \neq 0,-1,-2, \ldots$ It was proved that this neutrix limit exists for all $x, y$ and $p, q$ and so was used to define $B(x, y)$ for all $x, y$ and $p, q=0,-1,-2, \ldots$.

The following theorem was proved in [3].

\section{Theorem 1.}

$$
B_{p, q}(\lambda, \mu)=B_{q, p}(\mu, \lambda)
$$

for $p, q=0,1,2, \ldots$ and all $\lambda, \mu$.

The following results were also proved in [3].

$$
\begin{gathered}
B_{p, 0}(0,1)=0: \quad p=1,2, \ldots \\
B_{p, 0}(0,0)=B_{p, 0}(1,0)=(-1)^{p} p ! \zeta(p+1): \quad p=1,2, \ldots,
\end{gathered}
$$

where $\zeta$ denotes the zeta function.

$$
\begin{aligned}
B_{p, 0}(0, r+1) & =\sum_{i=1}^{r}\left(\begin{array}{l}
r \\
i
\end{array}\right) \frac{(-1)^{p+i} p !}{i^{p+i}}: \quad p, r=1,2, \ldots \\
B_{p, 0}(-1,0) & =-p !+(-1)^{p} \zeta(p+1): \quad p=1,2, \ldots \\
B_{p, 0}(-r-1,1) & =-\frac{p !}{(r+1)^{p+1}}: \quad p, r=0,1,2, \ldots
\end{aligned}
$$




\section{MAIN RESUltS}

We now prove the following generalization of equation (6).

\section{Theorem 2.}

$$
B_{p, 0}(-r, 0)=-\sum_{i=0}^{r-1} \frac{p !}{(r-i)^{p+1}}+(-1)^{p} p ! \zeta(p+1),
$$

for $p, r=1,2, \ldots$

Proof. We have

$$
\int_{\epsilon}^{1-\epsilon} t^{-r-1} \ln ^{p} t(1-t)^{-1} d t=\int_{\epsilon}^{1-\epsilon} t^{-r} \ln ^{p} t\left[t^{-1}+(1-t)^{-1}\right] d t
$$

and it follows that

$$
\begin{aligned}
B_{p, 0}(-r, 0) & =\mathrm{N}-\lim _{\epsilon \rightarrow 0} \int_{\epsilon}^{1-\epsilon} t^{-r-1} \ln ^{p} t(1-t)^{-1} d t \\
& =B_{p, 0}(-r, 1)+B_{p, 0}(-r+1,0) \\
& =-\frac{p !}{r^{p+1}}+B_{p, 0}(-r+1,0),
\end{aligned}
$$

on using equation ( 7 ) for $r, p=1,2, \ldots$

Now assume that equation (8) holds for some $r$ and $p=1,2, \ldots$ This is true when $r=1$ and $p=1,2, \ldots$ by equation (4). Then using equation (9) and our assumption, we have

$$
\begin{aligned}
B_{p, 0}(-r-1,0) & =-\frac{p !}{(r+1)^{p+1}}-\sum_{i=0}^{r-1} \frac{p !}{(r-i)^{p+1}}+(-1)^{p} p ! \zeta(p+1) \\
& =-\sum_{i=0}^{r} \frac{p !}{(r-i)^{p+1}}+(-1)^{p} p ! \zeta(p+1)
\end{aligned}
$$

and so equation (8) holds for $r+1$. Equation (8) now follows by induction.

\section{Theorem 3.}

$$
B_{p+1,0}(2,-1)=(-1)^{p+1}(p+1) ![\zeta(p+2)-\zeta(p+1)]
$$

for $p=1,2, \ldots$ and

$$
B_{1,0}(2,-1)=\zeta(2)-1
$$


Proof. We have

$$
\begin{aligned}
\int_{\epsilon}^{1-\epsilon} \ln ^{p} t(1-t)^{-1} d t & =\frac{1}{p+1} \int_{\epsilon}^{1-\epsilon} t(1-t)^{-1} d \ln ^{p+1} t \\
& =\frac{1}{p+1}\left[(1-\epsilon) \epsilon^{-1} \ln ^{p+1}(1-\epsilon)-\epsilon(1-\epsilon)^{-1} \ln ^{p+1} \epsilon\right] \\
& -\frac{1}{p+1} \int_{\epsilon}^{1-\epsilon}\left[(1-t)^{-1} \ln ^{p+1} t+t(1-t)^{-2} \ln ^{p+1} t\right] d t
\end{aligned}
$$

and it follows that

$$
\begin{aligned}
B_{p, 0}(1,0) & =\mathrm{N}-\lim _{\epsilon \rightarrow 0} \int_{\epsilon}^{1-\epsilon} \ln ^{p} t(1-t)^{-1} d t \\
& =\frac{1}{p+1} \mathrm{~N}-\lim _{\epsilon \rightarrow 0}\left[(1-\epsilon) \epsilon^{-1} \ln ^{p+1}(1-\epsilon)-\epsilon(1-\epsilon)^{-1} \ln ^{p+1} \epsilon\right] \\
& -\frac{1}{p+1} \mathrm{~N}-\lim _{\epsilon \rightarrow 0} \int_{\epsilon}^{1-\epsilon}\left[(1-t)^{-1} \ln ^{p+1} t+t(1-t)^{-2} \ln ^{p+1} t\right] d t \\
& =0-\frac{1}{p+1}\left[B_{p+1,0}(1,0)+B_{p+1,0}(2,-1)\right],
\end{aligned}
$$

for $p=1,2, \ldots$.

Using equation (4), we now have

$$
(-1)^{p} p ! \zeta(p+1)=(-1)^{p} p ! \zeta(p+2)-\frac{1}{p+1} B_{p+1,0}(2,-1)
$$

and equation (10) follows for $p=1,2, \ldots$.

In the particular case when $p=0$, we have

$$
\begin{aligned}
\int_{\epsilon}^{1-\epsilon}(1-t)^{-1} d t= & \int_{\epsilon}^{1-\epsilon} t(1-t)^{-1} d \ln t \\
= & {\left[(1-\epsilon) \epsilon^{-1} \ln (1-\epsilon)-\epsilon(1-\epsilon)^{-1} \ln \epsilon\right] } \\
& -\int_{\epsilon}^{1-\epsilon}\left[(1-t)^{-1} \ln t+t(1-t)^{-2} \ln t\right] d t
\end{aligned}
$$

and it follows that

$$
\begin{aligned}
B(1,0)= & \mathrm{N}-\lim _{\epsilon \rightarrow 0} \int_{\epsilon}^{1-\epsilon} \ln t(1-t)^{-1} d t \\
= & \mathrm{N}-\lim _{\epsilon \rightarrow 0}\left[(1-\epsilon) \epsilon^{-1} \ln (1-\epsilon)-\epsilon(1-\epsilon)^{-1} \ln \epsilon\right] \\
& -\mathrm{N}-\lim _{\epsilon \rightarrow 0} \int_{\epsilon}^{1-\epsilon}\left[(1-t)^{-1} \ln t+t(1-t)^{-2} \ln t\right] d t \\
= & -1-\left[B_{1,0}(1,0)+B_{1,0}(2,-1)\right] \\
= & -1+\zeta(2)-B_{1,0}(2,-1)=0
\end{aligned}
$$


on using equations (1) and (4), proving equation (11).

\section{Theorem 4.}

$$
B_{p, 0}(r, 0)=(r-1) B_{p, 1}(r-1,1)+p B_{p-1,1}(r-1,1),
$$

for $p=1,2, \ldots$ and $r=2,3, \ldots$.

Proof. Note that $B_{p, 1}(r-1,1)$ and $B_{p-1,1}(r-1,1)$ are standard forms of the Beta function.

We have

$$
\begin{aligned}
\int_{\epsilon}^{1-\epsilon} t^{r-1} \ln ^{p} t(1-t)^{-1} d t & =-\int_{\epsilon}^{1-\epsilon} t^{r-1} \ln ^{p} t d \ln (1-t) \\
& =-(1-\epsilon)^{r-1} \ln ^{p}(1-\epsilon) \ln \epsilon+\epsilon^{r-1} \ln ^{p} \epsilon \ln (1-\epsilon) \\
& +\int_{\epsilon}^{1-\epsilon}\left[(r-1) t^{r-2} \ln ^{p} t+p t^{r-2} \ln ^{p-1} t\right] \ln (1-t) d t
\end{aligned}
$$

and it follows that

$$
\begin{aligned}
B_{p, 0}(r, 0) & =\mathrm{N}-\lim _{\epsilon \rightarrow 0} \int_{\epsilon}^{1-\epsilon} t^{r-1} \ln ^{p} t(1-t)^{-1} d t \\
& =(r-1) B_{p, 1}(r-1,1)+p B_{p-1,1}(r-1,1),
\end{aligned}
$$

proving equation (12)

In the next theorem, the constants $c_{p, r}(i)$ are defined by the expansion

$$
(1-\epsilon)^{r-1} \ln ^{p}(1-\epsilon)=\sum_{i=1}^{\infty} c_{p, r}(i) \epsilon^{i}
$$

for $p, r=1,2, \ldots$ In particular

$$
c_{p, r}(s)=\left\{\begin{array}{cc}
0, & s<p \\
(-1)^{s}, & s=p, \\
r-s, & s=p+1 .
\end{array}\right.
$$

\section{Theorem 5.}

$$
B_{p, 0}(r,-s)=(r-1) B_{p, 0}(r-1,-s+1)+p B_{p-1,0}(r-1,-s+1)+\frac{c_{p, r}(s)}{s},
$$

for $p, s=1,2, \ldots$ and $r=2,3, \ldots$

In particular

$$
B_{p, 0}(r,-s)=(r-1) B_{p, 0}(r-1,-s+1)+p B_{p-1,0}(r-1,-s+1),
$$

for $s=1,2, \ldots, p-1$ and $p, r=2,3, \ldots$,

$$
B_{s, 0}(r,-s)=(r-1) B_{s, 0}(r-1,-s+1)+s B_{s-1,0}(r-1,-s+1)+\frac{(-1)^{s}}{s},
$$


for $s=1,2, \ldots$ and $r=2,3, \ldots$ and

$$
\begin{aligned}
& B_{s-1,0}(r,-s)=(r-1) B_{s-1,0}(r-1,-s+1) \\
& \quad+(s-1) B_{s-2,0}(r-1,-s+1)-\frac{(-1)^{s}(r-s)}{s},
\end{aligned}
$$

for $s=2,3, \ldots$ and $r=2,3, \ldots$.

Proof. We have

$$
\begin{aligned}
\int_{\epsilon}^{1-\epsilon} t^{r-1} \ln ^{p} t(1-t)^{-s-1} d t & =\frac{1}{s} \int_{\epsilon}^{1-\epsilon} t^{r-1} \ln ^{p} t d(1-t)^{-s} \\
& =\frac{(1-\epsilon)^{r-1} \ln ^{p}(1-\epsilon)}{s \epsilon^{s}}+\frac{\epsilon^{r-1} \ln ^{p} \epsilon}{s(1-\epsilon)^{s}} \\
+\int_{\epsilon}^{1-\epsilon} & {\left[(r-1) t^{r-2} \ln ^{p} t+p t^{r-2} \ln ^{p-1} t\right](1-t)^{-s} d t }
\end{aligned}
$$

and it follows that

$$
\begin{aligned}
& B_{p, 0}(r,-s)=\mathrm{N}-\lim _{\epsilon \rightarrow 0} \int_{\epsilon}^{1-\epsilon} t^{r-1} \ln ^{p} t(1-t)^{-s-1} d t \\
& \quad=\frac{c_{p, r}(s)}{s}+0+(r-1) B_{p, 0}(r-1,-s+1)+p B_{p-1,0}(r-1,-s+1),
\end{aligned}
$$

proving equation (14).

Equations follow (15) to (17) on using equation (13).

Corollary 5.1. $B_{p, 0}(r,-s)$ is a linear sum of the standard forms of the Beta function $B_{i, 0}(j, 1)$ for $s=1,2, \ldots$ and $p, r=s+2, s+3, \ldots$.

In particular,

$$
\begin{aligned}
B_{p, 0}(r,-1)= & (r-1)(r-2) B_{p, 0}(r-2,1) \\
& +p(2 r-3) B_{p-1,0}(r-2,1)+p(p-1) B_{p-2,0}(r-2,1)
\end{aligned}
$$

Proof. Equation (12) shows that the Corollary is true when $s=0$.

Now assume the Corollary is true for some positive integer $s$. We then have from equation (14) on noting that $c_{p, r}(s)=0$, since $p>s$,

$$
\begin{aligned}
& B_{p, 0}(r,-s-1)=(r-1) B_{p, 0}(r-1,-s)+p B_{p-1,0}(r-1,-s) \\
&=(r-1)\left[B_{p, 0}(r-1,-s+1)+p B_{p-1,0}(r-1,-s+1)\right] \\
&+p\left[(r-1) B_{p-1,0}(r-1,-s+1)+(p-1) p B_{p-2,0}(r-1,-s+1)\right]
\end{aligned}
$$

and it follows that the Corollary holds for $s+1$. The result now follows by induction. 
When $s=1$, we have from equation (15)

$$
\begin{aligned}
B_{p, 0}(r,-1)= & (r-1) B_{p, 0}(r-1,0)+p B_{p-1,0}(r-1,0) \\
= & (r-1)\left[(r-2) B_{p, 0}(r-2,1)+p B_{p-1,0}(r-2,1)\right] \\
& +p\left[(r-2) B_{p-1,0}(r-2,1)+(p-1) B_{p-2,0}(r-2,1)\right]
\end{aligned}
$$

and equation (18) follows.

\section{Theorem 6.}

$$
B_{p, 1}(0,1)=(-1)^{p+1} p ! \zeta(p+2)
$$

for $p=1,2, \ldots$

Proof. We have

$$
\begin{aligned}
\int_{\epsilon}^{1-\epsilon} t^{-1} \ln ^{p} t \ln (1-t) d t= & \frac{1}{p+1} \int_{\epsilon}^{1-\epsilon} \ln (1-t) d \ln ^{p+1} t \\
= & \frac{1}{p+1} \ln ^{p+1}(1-\epsilon) \ln \epsilon-\ln ^{p+1} \epsilon \ln (1-\epsilon) \\
& +\frac{1}{p+1} \int_{\epsilon}^{1-\epsilon}(1-t)^{-1} \ln ^{p+1} t d t
\end{aligned}
$$

and it follows that

$$
\begin{aligned}
B_{p, 1}(0,1) & =\mathrm{N}-\lim _{\epsilon \rightarrow 0} \int_{\epsilon}^{1-\epsilon} t^{-1} \ln ^{p} t \ln (1-t) d t \\
& =0+\frac{1}{p+1} B_{p+1,0}(1,0),
\end{aligned}
$$

and equation (19) follows on using equation (4).

Acknowledgement. The author would like to the thank the referee for his help in the improvement of this paper.

\section{REFERENCES}

[1] J.G. van der Corput, Introduction to the neutrix calculus, J. Analyse Math., 7 (195960), 291-398.

[2] B. Fisher and Y. Kuribayashi, Neutrices and the Beta function, Rostock. Math. Kolloq., 32 (1987), 56-66.

[3] E. Özçā̄ and B. Fisher, On partial derivatives of the Beta function, Rostock. Math. Kolloq., 45 (1991), 43-56.

[4] I.N. Sneddon, Special Functions of Mathematical Physics and Chemistry, Oliver and Boyd, Edinburgh and London, 1956. 
(Received: July 25, 2011)

(Revised: November 24, 2012)
Department of Mathematics University of Leicester Leicester, LE1 7RH, U.K.

E-mail: fbr@le.ac.uk 